\title{
Application of an Encoding Revision Algorithm in Overlapping Coalition Formation
}

\author{
Haixia Gui, Anhui University of Science and Technology, Huainan, China \\ Banglei Zhao, Anhui University of Science and Technology, Huainan, China \\ Huizong Li, Nanyang Normal University, Nanyang, China \\ Wanliu Che, Anhui University of Science and Technology, Huainan, China
}

\begin{abstract}
Overlapping coalition formation is a very active research field in multi-agent systems (MAS). In overlapping coalition, each agent can participate in different coalitions corresponding to multiple tasks at the same time. As each agent has limited resources, resource conflicts will occur. In order to resolve resource conflicts, the authors develop an improved encoding revision algorithm in this paper that can revise an invalid two-dimensional binary encoding into a valid one by checking the encoding for each row. To verify the effectiveness of the algorithm, differential evolution was used as the experimental platform and compared with Zhang et al. The experimental results show that the algorithm in this paper is superior to Zhang et al. in both solution quality and encoding revision time.
\end{abstract}

\section{KEYWORDS}

Differential Evolution, Encoding Revision, Multi-Agent System, Overlapping Coalition Formation, Resource Conflicts

\section{INTRODUCTION}

Distributed intelligent control based on multi-agent system (MAS) is booming(Bristow et al., 2014; Liao et al., 2014; Loia \& Vaccaro, 2014; Service \& Adams, 2011a; Ye et al., 2013), which is another leap in the development of control science. The coordination and cooperation between agents is the key issue of the control. In MAS, when an agent with limited resources meets with some tasks that could not be fulfilled, it will have to make interactive cooperation with other agents in the system for making a team to finish tasks mutually. Such a team is called a coalition(Guerrero \& Oliver, 2012; Ke et al., 2014; Liu et al., 2013).Coalition formation is the base of all activities in MAS, which will increase the effectiveness of individual agent to accomplish the tasks(He et al., 2017; Rahwan et al., 2012). Therefore, forming effective coalitions is a hot topic in the field of MAS. For instance, coalition formation has been successfully and widely applied to some fields like virtual enterprise $(\mathrm{He}$ et al., 2014; Huang et al., 2011; Zhang et al., 2010), wireless communications (Duarte et al., 2012; Khan et al., 2012; Schreiber \& Carley, 2013; Wang et al., 2016; Xiao et al., 2015), multi-robot cooperation(Dutta et al., 2014; Vig \& Adams, 2006), resource dispatch and allocation(Seow \& Sim, 2007), and combination optimization(Kulkarni \& Tai, 2010), etc. Generally speaking, most of the current research is about non-overlapping coalition(Service \& Adams, 2011b; Voice et al., 2014; 
Ye et al., 2015), in which an agent can join in only one coalition at any time. It means that once the coalition has been established, any agent who has joined in this coalition cannot join in other coalitions although the agent has more resources. However, one agent with enough resources should not be restricted to one coalition, instead, the agent can join in other coalitions to gain more profits, which is very common in virtual enterprises. Some small enterprises with sufficient resources can join in several coalitions to gain more reward through providing services. Apparently, this overlap can increase the utilization of agents resources and the efficiency of tasks fulfillment and the profit of the whole system, which is very popular among wireless network field(Wang et al., 2014; Xu et al., 2016). Therefore, in order to increase resources utilization rate and tasks solving efficiency of the whole system overlapping coalition allows one agent to join in different coalitions to accomplish different tasks by allocating its resources. It is more suitable for the cases in the real world, improving the capability of a single agent to solve the problems in the tasks, and resulting in resource reorganization and complementing advantages mutually.

\section{RELATED WORK}

Overlapping coalition means that each agent joins in the executions of many tasks at the same time. A group of agents join in a series of tasks with the limited time or resources, and one agent for many tasks will cause resource conflicts because each agent's resources are limited. As mentioned before, most of the current studies are about non-overlapping coalition, while the overlapping coalition tends to be neglected. It has not been studied for a long time, and still in the process of exploration and research, leaving many problems unsolved.

For the first time, Shehory and Kraus(1996) propose a greedy algorithm for the overlapping coalition formation. This algorithm solves the possible coalition in the absence of limited resources, which has high communication costs and waste of resources. Chalkiadakis et al.(2010) explore OCF through the "Kernel" concept in game theory. But in this method it is assumed that the overlapping coalition does not exist under the conditions of resource conflict, which is obviously not consistent with the reality. Wilson and desJardins(2007) propose a solution of payoff distribution to form stable overlapping coalitions among self-interested and unreliable agents. Sen and Dutta(2000) adopt one-dimensional integral encoding genetic algorithm to search for the optimal coalition. Yang and Luo(2007) improve on Sen and Dutta's study and solve coalition generation based on two-dimensional binary encoding and corresponding revision algorithm. Lin and $\mathrm{Hu}(2007)$ put up with an encoding revision algorithm (henceforth called $\mathrm{LH}$ ), transferring all residual resources of effective coalition to a selected agent, which will carry out all the effective resources to help other coalition, thus avoiding resource conflicts. But it is assertive to judge whether the encoding is legal or not, which will cause many encodings that could have been modified to be discarded, resulting into the lower efficiency of algorithm. Xu and Li (2008)(henceforth called XL) transfer every dimensional capability of each agent to many different subagents, and adopt two-dimension binary encoding where a row denotes an agent and a column denotes a subagent. However, with this algorithm each encoding is not guaranteed as legal. Many invalid encodings will be discarded at each iteration. And in this algorithm, resource conflicts of overlapping coalition also have been neglected. Zhang et al. (2011) put forward overlapping coalition formation based on two-dimensional binary encoding the particle swarm optimization (PSO) algorithm. Firstly, each line is checked through the random selection of agents with the appropriate resources to join the coalition to ensure that the coalition is feasible, and then each column is inspected to solve the problem of resource conflicts. For each column, check the " 1 " of the column with the minimum amount of contribution in the coalition. If the agent represented in the column cannot satisfy the request of multiple tasks at the same time, it must secede from the coalition in order to avoid resource conflicts. Then, these coalitions may be not feasible once more, other agents will be selected for the coalitions. But with this algorithm all rows and columns must be checked, with its operation being rather complex. To address these shortcomings, we apply two-dimensional binary 
encoding differential evolution algorithm into OCF. We provide an algorithm to modify an invalid encoding into a valid encoding without resource conflicts. Finally, we verify the performance of the algorithm by the experiment and compare it with the algorithm by Zhang et al. (2011)

\section{OVERLAPPING COALITION MODEL}

Suppose that there are $n$ agents in MAS, $A=\left\{a_{1}, \cdots, a_{n}\right\}$, which have to cooperate to fulfill $m$ tasks, $T=\left\{t_{1}, \cdots, t_{m}\right\}$.

Definition 1. $\forall a_{j} \in A, j \in\{1, \cdots, n\}$, has $r$ types of original resources $B_{j}=\left[b_{1}^{j}, b_{2}^{j}, \cdots, b_{r}^{j}\right]$, $0 \leq b_{k}^{j}<\infty, k=1, \cdots, r, r \in N$, where $b_{k}^{j}$ is the amount of resource of type $k$ held by agent $a_{j}$.

Definition 2. $\forall t_{i} \in T, i \in\{1, \cdots, m\}$, has $r$ types of demanded resources $D_{i}=\left[d_{1}^{i}, d_{2}^{i}, \cdots, d_{r}^{i}\right]$, $0 \leq d_{k}^{i}<\infty, k=1, \cdots, r, r \in N$, where $d_{k}^{i}$ is the amount of resource of type $k$ required by task $t_{i}$.

Definition 3. A coalition $C_{i}, C_{i} \subset A$ and $C_{i} \neq \varphi$, the coalition for $t_{i}$. In overlapping coalition, each agent can participate in multiple coalitions to contribute their resources at the same time. Thus, for each coalition $C_{i}, \forall a_{j} \in A$ has a real distribution resources $W_{j i}=\left[w_{1}^{j i}, w_{2}^{j i}, \cdots w_{r}^{j i}\right], 0 \leq w_{k}^{j i} \leq b_{k}^{j}$. Note that, if $a_{j}$ does not join in $C_{i}, W_{j i}=0$. Obviously, the sum of $a_{j}$ 's real distribution resources for all tasks should not exceed the initial total amount of its resources, otherwise it will generate resource conflicts. That is, in order to avoid resource conflicts, it must meet $\sum_{i=1}^{m} w_{k}^{j i} \leq b_{k}^{j}$. And the resources vector of $C_{i}$ is $B_{C_{i}}=\left[b_{1}^{C_{i}}, b_{2}^{C_{i}}, \cdots, b_{r}^{C_{i}}\right], b_{k}^{C_{i}} \geq 0$. It should be the sum of the actual contribution of the coalition's member, that is, $\forall k \in\{1, \cdots, r\}$, there is $b_{k}^{C_{i}}=\sum_{a_{j} \in C_{i}} w_{k}^{j i}$, namely, for requirement resources of the task, $b_{k}^{C_{i}}=\sum_{a_{j} \in C_{i}} w_{k}^{j i}=d_{k}^{i}$ is satisfied.

Definition 4. $\forall a_{j} \in A$ has $r$ types of residual resources $P_{j}=\left[p_{1}^{j}, p_{2}^{j}, \cdots, p_{r}^{j}\right], 0 \leq p_{k}^{j} \leq b_{k}^{j}$, it is the residual resources after $a_{j}$ has joined in some coalition. Note that, if $a_{j}$ does not join in any coalition, $P_{j}=B_{j}$, otherwise $p_{k}^{j}=b_{k}^{j}-\sum_{i=1}^{m} w_{k}^{j i}$.

Definition 5. The value of $C_{i}$ is calculated by a characteristic function $V\left(C_{i}\right) \geq 0$ (He et al., 2014; Huang et al., 2011), $V\left(C_{i}\right)=\Phi\left(t_{i}\right)-\Theta\left(C_{i}\right)-\Pi\left(C_{i}\right)$, where $\Phi\left(t_{i}\right)$ is a reward for performing $t_{i}$ and is usually a given constant number, $\Theta\left(C_{i}\right)$ is the total resource cost of all members in $C_{i}$, $\Pi\left(C_{i}\right)$ is the total communication cost between agents in $C_{i}$. Suppose the cost of the communication between $a_{j_{1}}$ and $a_{j_{2}}$ is $\xi_{j_{1}, j_{2}}$, which is generally a given constant number, satisfying $\xi_{j_{1}, j_{1}}=0$, $\xi_{j_{1}, \mathrm{j}_{2}}=\xi_{j_{2}, \mathrm{j}_{1}}$. If $C_{i}=\left\{a_{j_{1}}, a_{j_{2}}, a_{j_{3}}\right\}$, then $\varepsilon\left(C_{i}\right)=\xi_{j_{1}, \mathrm{j}_{2}}+\xi_{j_{1}, \mathrm{j}_{3}}+\xi_{j_{2}, \mathrm{j}_{3}}$.

In this way, OCF is just generating $m$ coalitions, $C_{1}, \cdots, C_{m}$, simultaneously according to task $t_{1}, \cdots, t_{m}$ under the formula (1). The object is to maximize the total profits of the system, namely satisfy the formula(2). 
$\sum_{j=1}^{n} b_{k}^{j} \geq \sum_{i=1}^{m} d_{k}^{i}, k=1, \cdots, r$

$v_{M A S}=\sum_{i=1}^{m} v\left(C_{i}\right)$

Among them, as in Formula (1), the initial capability of all agents should not be less than the ability demanded in all tasks.

\section{TWO-DIMENSION BINARY ENCODING}

The so-called two-dimension binary encoding is $0-1$ matrix of $m \times n$, where each row represents a task to be solved and each column represents an agent. The element at the intersection of row $i$ and column $j$ is defined as $\gamma_{i j} \cdot \gamma_{i j}=1$ means that agent $a_{j}$ will take part in the corresponding coalition $C_{i}$ to be solved for task $t_{i} \cdot \gamma_{i j}=0$ means that agent $a_{j}$ will not join in coalition $C_{i}$ to perform task $t_{i}$. Therefore, the agents with $\gamma_{i j}=1$ of each line in the encoding constitute a corresponding solving coalition for the task, as shown in Figure 1.

Figure 1. Two-dimensional binary encoding

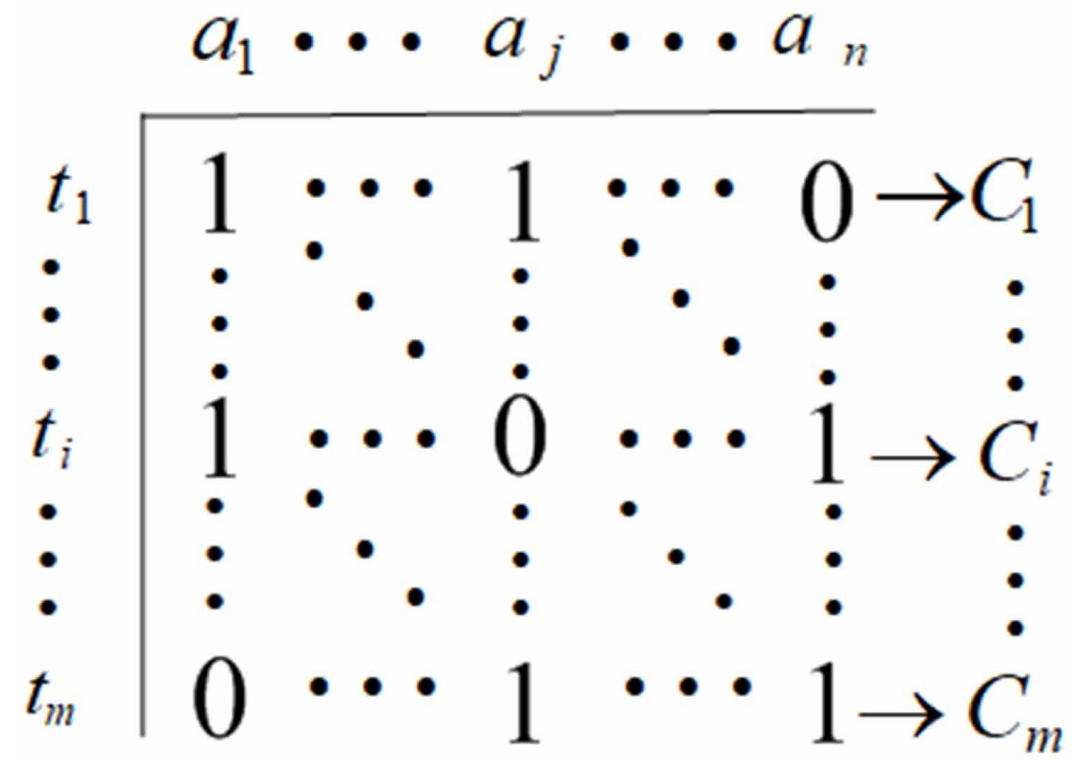

According to the definition of overlapping coalitions, each agent can join several coalitions corresponding to different tasks at the same time, so that there may be multiple " 1 " in each column of the above code. To ensure that this coding is legitimate, there are two aspects to consider: 
1. For each line in the code, it represents the corresponding solving coalition $C_{i}$ of each task. The resources owned by coalition $C_{i}$ cannot fulfill the requirements of task $t_{i}$, and then $C_{i}$ is an invalid coalition.

2. For each column in the code, each agent can join several different coalitions at the same time. However, due to the limited resources of each agent, it cannot meet the competition of multiple coalitions, so it will produce resource conflicts.

As long as any of the above situations occurs in the code, it will be an illegal code. Therefore, this paper proposes an encoding revision algorithm, which can correct any illegal code into a legal code.

\section{THE ENCODING REVISION ALGORITHM}

\subsection{The Description of the Algorithm}

The main idea of the algorithm: once an agent is selected to join a coalition, the number of resources the agent provides should be not greater than the resources values of the agent itself. Meanwhile, we calculate the resources it actually contributes to its coalition and its residual resources; then this agent can join other coalitions by using its residual resources. Note that, if an agent does not have any residual resources, it will not participate in any other tasks. The main steps of the algorithm are described as follows:

Step 1. First, initializing the valuesof $C_{i}, B_{C_{i}}, P_{j}$ and $W_{j i}$, namely, $C_{i} \leftarrow \phi, B_{C_{i}} \leftarrow 0, P_{j} \leftarrow B_{j}, W_{j i} \leftarrow 0$. Step 2. When selecting an uncheck row $i$ arbitrarily, we choose randomly an uncheck column $\mathrm{j}$ with $\gamma_{i j}=1$ in row $i$.

Step 2.1. If $\gamma_{i j}=1, B_{C_{i}}<D_{i}$ and $\min \left\{D_{i}-B_{C_{i}}, P_{j}\right\}>0$, the coalition $C_{i}$ can not accomplish the task $t_{i}$, then calculate $W_{j i}, W_{j i} \leftarrow \min \left\{D_{i}-B_{C_{i}}, P_{j}\right\} . W_{j i}$ is the lowest resources numbers contributed by $a_{j}$ to $C_{i}$. At the same time, calculate $a_{j}$ 's residual resources and $C_{i}$ 's distributed resources, that is $P_{j} \leftarrow P_{j}-W_{j i}, B_{C_{i}} \leftarrow B_{C_{i}}+W_{j i}$.

Step2.2. If $\gamma_{i j}=1, B_{C_{i}}<D_{i}$ and $\min \left\{D_{i}-B_{C_{i}}, P_{j}\right\}=0$, the coalition $C_{i}$ can not perform the task $t_{i}$, either. But $a_{j}$ does not have any resources to provide at the moment, and $a_{j}$ is made to leave $C_{i}$, namely, $\gamma_{i j} \leftarrow 0$.

Step 2.3. If $\gamma_{i j}=1, B_{C_{i}}=D_{i}$, it means that Coalition $C_{i}$ can perform the task $t_{i} ; a_{j}$ is not essential for $C_{i}$, and $a_{j}$ is made to drop out of $C_{i}$ by $\gamma_{i j} \leftarrow 0$. This is done for the purpose of reducing communication cost and increasing $C_{i}$ 's profit.

Repeat the above operation until all columns with $\gamma_{i j}=1$ in the row are checked.

Step 3. If all bits " 1 " in row $i$ can not cooperate to perform Task $t_{i}$, namely $B_{C_{i}}<D_{i}$, we can select randomly $a_{j^{*}}$ with $P_{j^{*}}>0$ from $A-C_{i}$ to join $C_{i}$ and carry out $\gamma_{i j^{*}} \leftarrow 1, C_{i} \leftarrow C_{i}+\left\{a_{j^{*}}\right\}$, $\mathrm{W}_{j^{*} i} \leftarrow \min \left\{D_{i}-B_{C_{i}}, P_{j^{*}}\right\} . W_{j^{*} i}$ is the resource numbers that $a_{j^{*}}$ should contribute at least for $C_{i}$. At the same time, $a_{j^{*}}$ 's residual resources and $C_{i}$ 's distributed resources are figured out, that is, $P_{j^{*}} \leftarrow P_{j^{*}}-W_{j^{*} i}, B_{C_{i}} \leftarrow B_{C_{i}}+W_{j^{*} i}$. Repeat the operation until $C_{i}$ can accomplish $t_{i}$, namely $B_{C_{i}}=D_{i}$. 


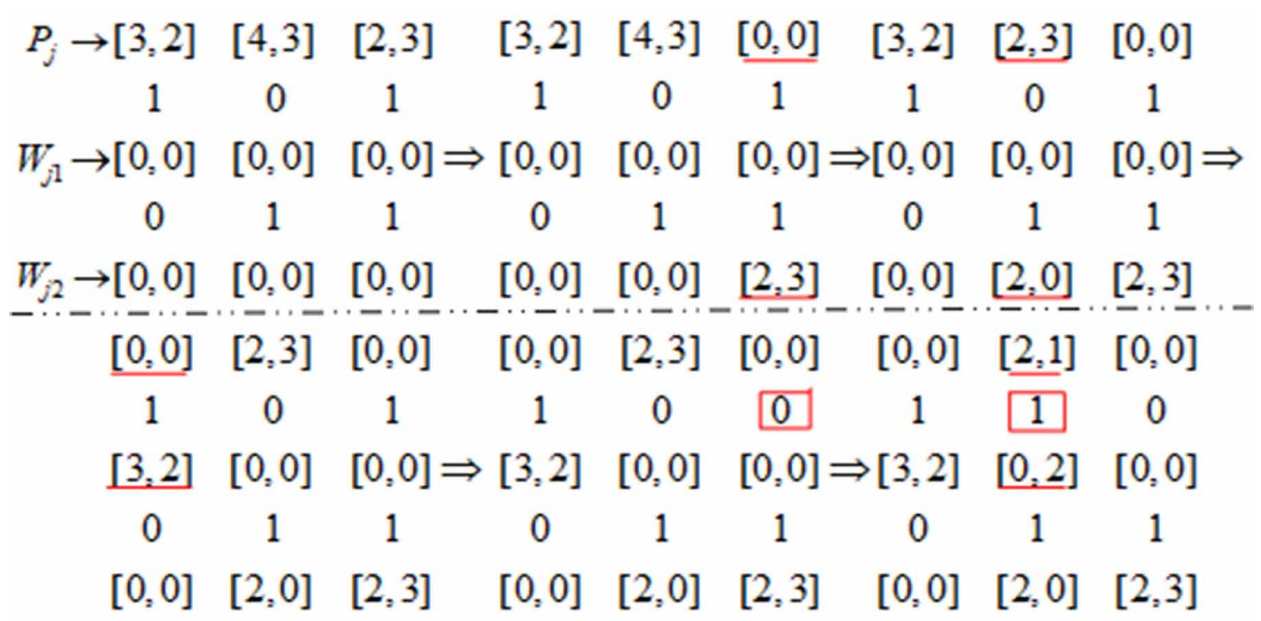

Step 4. Similarly, we select randomly unchecked rows revising encoding until all rows are checked, and then the algorithm is ended.

\subsection{Instances Specification}

For example, given $A=\left\{a_{1}, a_{2}, a_{3}\right\}$ and $T=\left\{t_{1}, t_{2}\right\}$ with $B_{1}=[3,2], B_{2}=[4,3], B_{3}=[2,3]$ and $D_{1}=[3,4], D_{2}=[4,3]$. The specific steps of encoding revision is illustrated in Figure 2 .

The specific revision process is as follows: Row 2 is arbitrarily selected to check, now $B_{C_{2}}=0<D_{2}$. In Row 2, Column 3 is stochastically chosen and $W_{32} \leftarrow \min \left\{D_{2}-B_{C_{2}}, P_{3}\right\}=[2,3]$, here $B_{C_{2}} \leftarrow[2,3], \quad P_{3} \leftarrow[0,0], \quad C_{2} \leftarrow\left\{a_{3}\right\}$. Then Column 2 is selected and $W_{22} \leftarrow \min \left\{D_{2}-B_{C_{2}}, P_{2}\right\}=[2,0]$, so $B_{C_{2}} \leftarrow[4,3], P_{2} \leftarrow[2,3], C_{2} \leftarrow\left\{a_{2}, a_{3}\right\}$. With this, Row 2 has been checked. Then Row 1 is selected, now $B_{C_{1}}=0<D_{1}$. In Row 1,Column 1 is randomly chosen and $W_{11} \leftarrow \min \left\{D_{1}-B_{C_{1}}, P_{1}\right\}=[3,2]$, here $B_{C_{1}} \leftarrow[3,2], P_{1} \leftarrow[0,0], C_{1} \leftarrow\left\{a_{1}\right\}$. Then Column 3 is selected but $a_{3}$ does not have any residual resource, so let $a_{3}$ drop out of $C_{1}$ and $\gamma_{13} \leftarrow 0$. However, $B_{C_{1}}<D_{1}$, thus $a_{2}$ with residual resources is chosen to join $C_{1}, \gamma_{12} \leftarrow 1$, $W_{21} \leftarrow \min \left\{D_{1}-B_{C_{1}}, P_{2}\right\}=[0,2], B_{C_{1}} \leftarrow[3,4], P_{2} \leftarrow[2,1], C_{1} \leftarrow\left\{a_{1}, a_{2}\right\}$. Thus, Row 1 has been checked. The encoding is revised to be valid.

\subsection{Performance Analysis}

Proposition 1. Any $m \times n$ invalid encoding can be revised to be valid when satisfying,Format(1) based on our algorithm.

PROOF We will argue by the mathematical induction.

Step1. There is only a task to be performed with $m=1$. Given constraint set (1), it is sure that the task can be completed without any resource conflict, that is, the proposition is true with $m=1$. 
Step2. Suppose that the proposition is true with $m=m^{\prime}$, the rows before $m^{\prime}$ being revised to be valid, namely, the former $m^{\prime}$ tasks can be accomplished, then the only Task $m$ need to be allocated resources, there is $\sum_{i=1}^{m^{\prime}} B_{C_{i}}=\sum_{i=1}^{m^{\prime}} D_{i}$.

Step3. When $m=m^{\prime}+1$, without losing the generality, we suppose that the tasks before $m^{\prime}$ can be performed from the induction hypothesis. Therefore, it is necessary to prove that Task $m^{\prime}+1$ can be fulfilled.

Given constraint set (1), we have $\sum_{j=1}^{n} B_{j} \geq \sum_{i=1}^{m} D_{i}$

For Task $m^{\prime}+1$, we have $\sum_{j=1}^{n} B_{j}-\sum_{i=1}^{m^{\prime}} B_{C_{i}} \geq \sum_{i=1}^{m} D_{i}-\sum_{i=1}^{m^{\prime}} D_{i}$. From the above, we can easily find that $\sum_{j=1}^{n} B_{j}-\sum_{i=1}^{m '} B_{C_{i}}$ represents just the residual resources of all agents after they have fulfilled the $m^{\prime}$ tasks, that is, $\sum_{j=1}^{n} B_{j}-\sum_{i=1}^{m^{\prime}} B_{C_{i}}=\sum_{j=1}^{n} P_{j}$, and $\sum_{i=1}^{m} D_{i}-\sum_{i=1}^{m^{\prime}} D_{i}$ is just the required resources of Task $m^{\prime}+1$, that is, $\sum_{i=1}^{m} D_{i}-\sum_{i=1}^{m^{\prime}} D_{i}=D_{m^{\prime}+1}$. Therefore, we have $\sum_{j=1}^{n} P_{j} \geq D_{m^{\prime}+1}$, that is Task $m^{\prime}+1$ can easily be satisfied with sufficient residual resources. To sum up, all $m$ tasks can be completed.

So the proposition is verified.

Proposition 2. Given an arbitrary OCF instance, the worst case complexity of our algorithm is $o(m \times n \times r)$.

PROOF. Judging from the algorithm in this paper, giving the value of each $W_{j i}$ needs to consider all types of resources, so the number of operations required to initialize is $o(m \times n \times r)$. Moreover, there are up to $m$ rows which should be checked. Once Row $i$ is selected, we at most need to examine $n$ agents for revising encoding, and each agent has up to $r$ types of resources. The complexity of this operation in the coalition $C_{i}$ is $o(n \times r)$. The number of operations required to check all rows is $o(m \times n \times r)$.

To sum up, the worst case complexity of the algorithm in this paper is $o(m \times n \times r)$.

\section{OVERLAPPING COALITION FORMATION BASED ON DIFFERENTIAL EVOLUTION}

\subsection{Differential Evolution}

Differential evolution (DE) is an evolutionary algorithm based on population differences by Storn and Prince in 1995(1995), and it is a kind of algorithm based on real number coding and explores the search space by three basic operations: mutation, crossover and selection(Storn \& Price, 1997). In the process of evolution, the mutation operation and the crossover operation are used to generate new trial individuals. The selection operation generates the next generation population by comparing the fitness values between the current evolutionary individuals and the trial individuals. Although it is simple and has few control parameters, DE has good convergence and has been successfully applied in many scientific and engineering fields(Cavalini et al., 2016; Dhaliwal, 2017; Panigrahi, 2017; Yu, 2017; Zhao et al., 2015). The operation process about the standard differential evolution will be described as follows. 


\subsubsection{Mutation}

The mutation operation is performed by individual vector differences. Suppose $x_{u}(t)$ to be the current evolutionary individual, $u$ is the serial number of the current individual in the population, $t$ is the evolutionary algebra. Select randomly three individuals from the populations, $x_{s 1}(t), x_{s 2}(t)$ and $x_{s 3}(t)$, with $\mathrm{s} 1 \neq s 2 \neq s 3 \neq u$, then the mutation operation is defined as follows:

$$
h_{u}(t+1)=x_{s 1}(t)+\lambda\left(x_{s 2}(t)-x_{s 3}(t)\right)
$$

Among them, $\lambda$ is a scaling factor (with $\lambda \in[0,2]$ ) to control the scaling degree of difference vector.

\subsubsection{Crossover}

The trial individual $g_{u}(t+1)$ is generated by crossing the current evolutionary individual $x_{u}(t)$ with its mutation individual $h_{u}(t+1)$. The widely used binomial crossover is defined as the following equation:

$g_{u v}(t+1)=\left\{\begin{array}{l}h_{u v}(t+1), \operatorname{randf}_{u v}(0,1) \leq C R \text { or } v=\operatorname{randi}(1, D) \\ x_{u v}(t), \operatorname{randf}_{u v}(0,1)>C R \text { or } v \neq \operatorname{randi}(1, D)\end{array}\right.$

Among them, $\operatorname{randf}_{u v}(0,1)$ is a uniformly distributed random number within $(0,1)$,

randi $(1, D)$ is a random integer within $\{1,2, \cdots, D\}, C R$ is the crossover probability (with $C R \in(0,1))$.

\subsubsection{Selection}

The trial individual $g_{u}(t+1)$ replaces the current evolutionary individual $x_{u}(t)$ if its fitness value is better. Otherwise, the current evolutionary individual is reserved in the next generation. The selection operator is defined as the following equation:

$x_{u}(t+1)=\left\{\begin{array}{l}g_{u}(t+1), f\left(g_{u}(t+1)\right) \leq f\left(x_{u}(t)\right) \\ x_{u}(t), f\left(g_{u}(t+1)\right)>f\left(x_{u}(t)\right)\end{array}\right.$

Through the operations of mutation, crossover and selection, each individual in the initial population is cycled to obtain the next generation population, which evolves several generations to get the optimal solution of the problem. The scaling factor $\lambda$ and the crossover probability $C R$ in the differential evolution algorithm are used to determine the optimal combination by experimental method.

\subsection{Description of the Algorithm for Overlapping Coalition Formation based on Differential Evolution}

At present, the research and application of differential evolution are generally expressed by real number encoding. In this paper, binary encoding is proposed to solve the overlapping coalition formation. The result of the mutation operation is real number in the standard differential evolution, but the individual is a binary encoding in this paper, so the results of the mutation operation are no longer the feasible solution to the problem. In this way, we make appropriate adjustment about the 
mutation operation, thus the function $\operatorname{Sig}(x)$ is introduced, which is a sigmoid limiting transformation function, satisfying

$\operatorname{Sig}(x)=1 /(1+\exp (-x))$.

Here, $x$ is the result of the mutation operation performed by the standard differential evolution. The mutation operation is adjusted to as follows:

$h_{u}{ }^{\prime}(t+1)=\left\{\begin{array}{l}1, \operatorname{Sig}(x)>\operatorname{rand}() *\left(1 / R A N D_{-} M A X\right) \\ 0, \text { otherwise }\end{array}\right.$

Where $R A N D \_M A X$ is the largest random number and $\operatorname{rand}()$ is the number between 0 and the largest random number.

The steps of the algorithm for overlapping coalition formation based on differential evolution:

Step 1. Initialize binary encoding and generate randomly the first generation population.

Step 2. Revise every individual's encoding of the initial population by the encoding revision algorithm. Step 3. Firstly, perform mutation and crossover operations on the revised initial population. Then, revise every individual's encoding and calculate its fitness based on (2) to form trial population. Finally, compare the two groups of population and execute selection operation to create the next generation. If the generation number (which can be denoted as GN) is not reached, repeat the above steps to continue the evolution of every individual.

Step 4. Output the results.

The flow chart of the proposed algorithm is shown in Figure 3.

\section{EXPERIMENTAL RESULTS AND ANALYSIS}

In order to evaluate the effectiveness of the proposed algorithm, experimental simulation is carried out and the results are compared. Given the number of agents $n=10$, the number of tasks $m=4$, and the number of resource types $r=2$, we use the algorithm from (Zhang et al., 2011) and ours respectively to solve the overlapping coalition formation problem and compare their results. For the algorithm of Zhang et al. (2011), the particle swarm optimization (PSO) algorithm is adopted as the experiment platform, with the particle number being 20 and the maximum particle speed being 6.0. For implementing our algorithm, we use the differential evolution algorithm as the experiment platform, where the crossover possibility is 0.9 , the scaling factor is 0.6 and the population size is also 20 . The maximum iteration number is 500 for both algorithms which run 50 times respectively. Due to different platforms of the two algorithms, for the sake of fairness, the comparison is made in terms of encoding revision time rather than the total time consumed. Experiments are conducted under the following two settings:

1. The resources owned by all the agents are equal to those required by all the tasks, i.e.

$$
\sum_{j=1}^{n} b_{k}^{j}=\sum_{i=1}^{m} d_{k}^{i}, k=1, \cdots, r \text {. }
$$


International Journal of Cognitive Informatics and Natural Intelligence

Volume 15 • Issue 4 • October-December 2021

Figure 3. The flow chart of the proposed algorithm

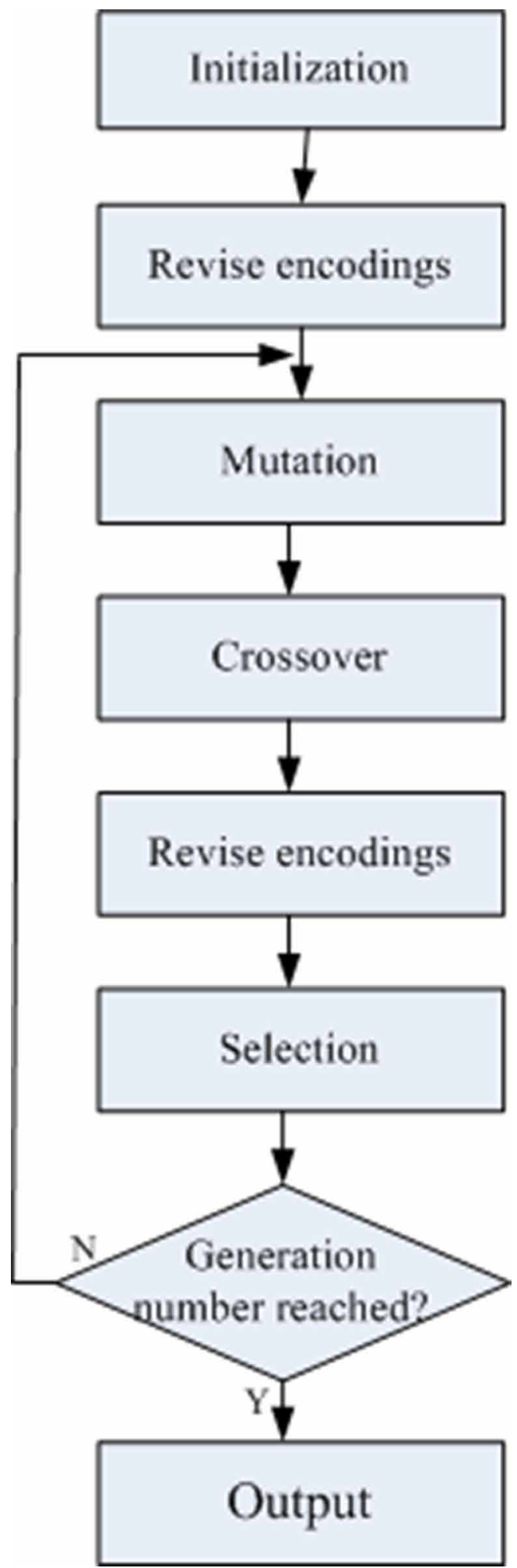


2. The resources owned by all the agents are larger than those required by all the tasks, i.e. $\sum_{j=1}^{n} b_{k}^{j}>\sum_{i=1}^{m} d_{k}^{i}, k=1, \cdots, r$.

Figure 4 shows the total profits of the two algorithms for every solution under two experimental settings. From the figures, we can see that our algorithm outperforms that of Zhang et al. (2011) under both settings, and the total profits under the second setting are better than those under the first. This is because in the proposed revision algorithm, when a coalition $C_{i}$ is feasible, the redundant agents will be removed from the coalition, i.e. the corresponding " 1 " will be changed to " 0 ". But for the algorithm of Zhang et al., (2011) changing from " 1 " to " 0 " would cause the infeasibility of $C_{i}$ and thus other agents need to be chosen and added to the coalition to finish the task. Therefore, it is impossible for the algorithm of Zhang et al. (2011) to reduce its size of the coalition $C_{i}$, leading to inferior total system profits to ours. However, in the second setting, each agent owns more sufficient resources than in the first setting, thus to accomplish each task needs fewer agents, which means reduced communication costs and leads to better total profits compared with the first setting.

Figure 5 demonstrates the encoding revision time of the two algorithms for each experiment under the two experimental settings. From the figures, it can be seen that our algorithm is better than that of Zhang et al. (2011) under both settings, and the overall encoding revision time of the second setting is a little better than that of the first. This can be explained as follows. Our proposed revision algorithm randomly chooses a row for encoding revision, and then other random rows, i.e. only checking all rows, which greatly reduces the time cost. Comparatively, the algorithm by Zhang et al. (2011) first chooses a row, and then checks all the corresponding columns, till all rows are checked, i.e. checking all rows and columns. In the second setting, since each agent has more sufficient resources than the case in the first setting, the adaption times is less than that in the first setting, which means slightly better encoding revision time than that in the first setting.

To further validate the performance of our proposed algorithm, experiments have also been conducted with different parameters including the numbers of agents, tasks, and types of resources, with each run for 30 times and average value of the performance measured.

(1) Variation in the number of agents

We first test the performance of the two algorithms with respect to the number of agents $n$ increasing from 16 to 30 at the number of tasks $m=4$ and the number of resource types $r=2$. Figure 6 shows the average total profits of the two algorithms with different $n$ for two experimental settings. In the first setting, the profit of our algorithm is obviously better than that of Zhang et al. (2011) The performance of both algorithms drops obviously as $n$ increases. In the second setting, the two algorithms do not show much profit difference. This is because under the first setting, the sum of resources owned by all agents is fixed. As $n$ increases, the resources owned by each agent will decrease, which results in an increased number of agents required to accomplish each task, i.e. more members in the coalition, which will take more communication costs between agents and reduce the coalition value, i.e. gradually decreasing the total profits. However, under the second setting, the agents have sufficient resources and it only needs part of the agents to accomplish the task. With the increasing of $n$, both algorithms tend to select those agents with relatively low communication costs into the coalition for task accomplishment, leading to increased system profits, thus the total profit in Figure 6(b) is overall better than that in Figure 6(a). In cases where agents have enough resources, our algorithm and that of (Zhang et al., 2011) accomplish each task with the fixed coalition, thus there is little difference in the total system profits. 
Figure 4. The total profits of each trial

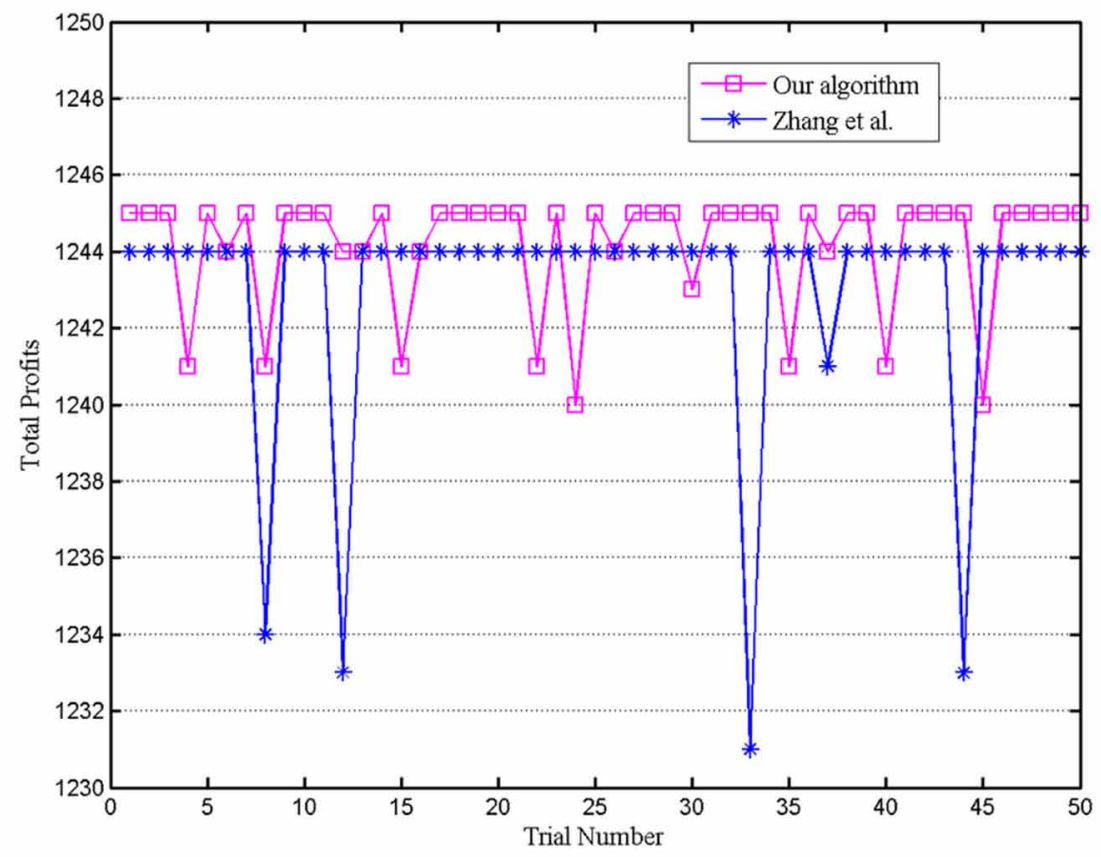

Figure 5.The encoding revision time of each trial

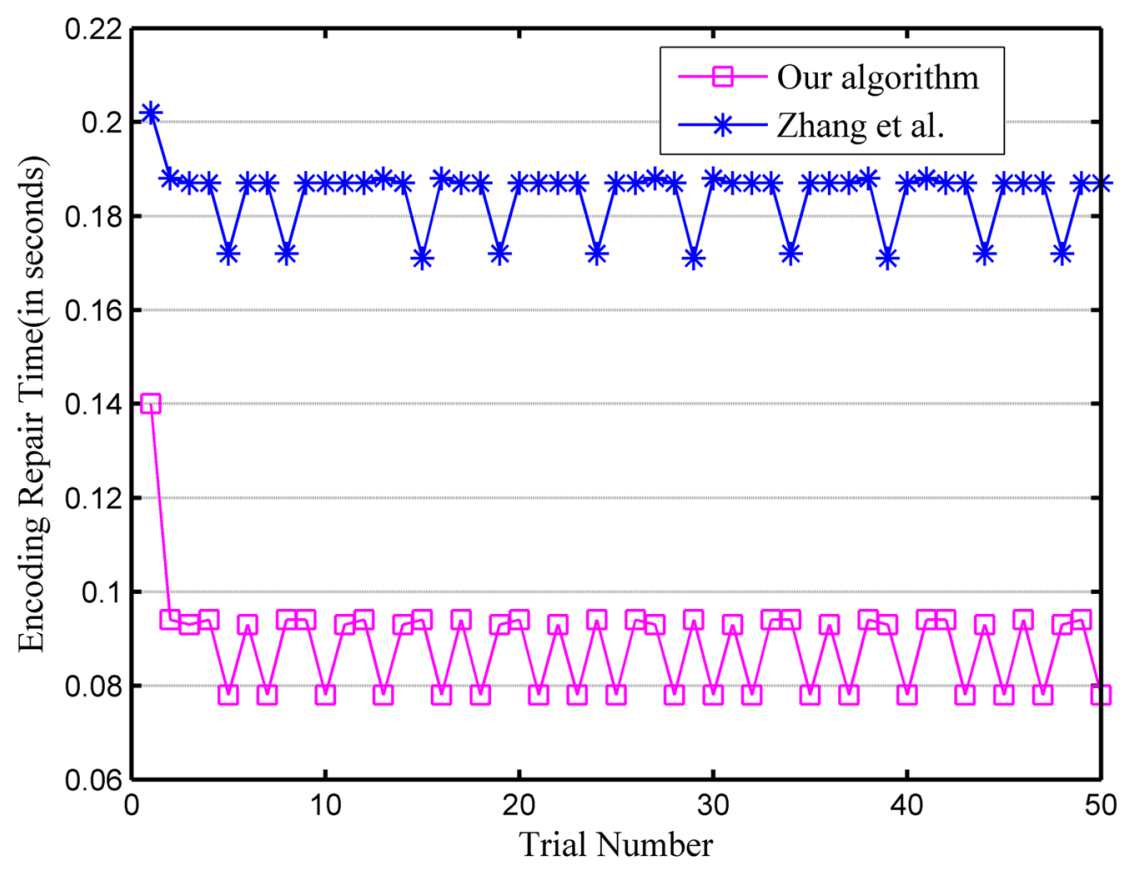


Figure 6. The average total profits of each algorithm with different $n$

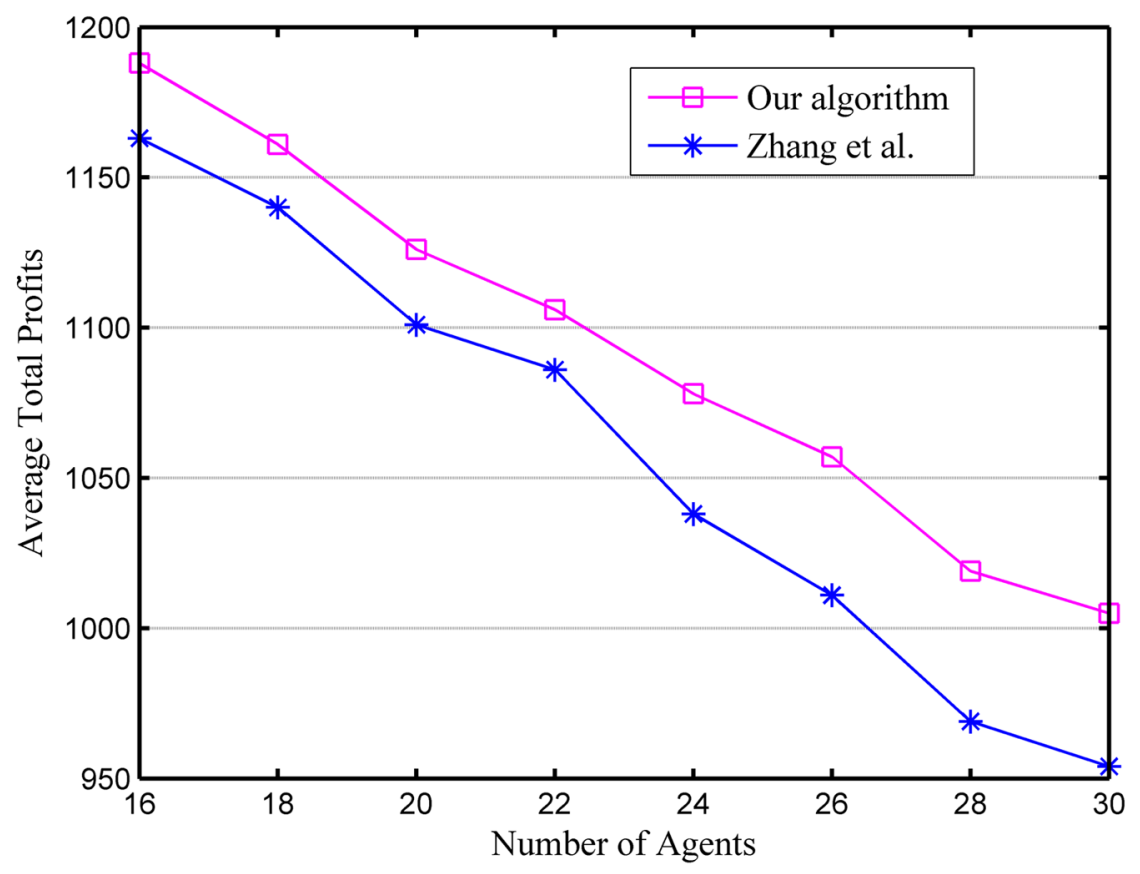

Figure 7 shows the average encoding revision time of the two algorithms with different $n$ under two experimental settings, from which we can see it is better in the second setting than in the first. With the increasing of $n$, the average encoding revision time of both algorithms will grow under two experiments settings, but generally our algorithm performs better than that of Zhang et al. (2011), with a larger margin for a bigger $n$, which indicates that the latter is more influenced by $n$. This is because their algorithm needs to check every column to deal with the resource conflicts, and with the increasing of $n$, many repetitive and useless operations are performed, leading to huge time costs of the algorithm. As $n$ increases, the resources owned by each agent will reduce while those required by each task remain unchanged, thus more encoding revision operations are needed, leading to increased encoding revision time.

(2) Variation in the number of tasks

Then we test the performance of the two algorithms with respect to the number of tasks. The number of tasks $m$ changes from 6 to 20, the number of agents $n=10$, and the number of resource types $r=2$. Figure 8 shows the average total profits of the two algorithms at different $m$ under the two experimental settings respectively. It can be observed from the figures that, in the first setting, our algorithm performs slightly higher than that of Zhang et al. (2011); in the second setting, the two algorithms reveal similar performance. But under either setting, with the increasing of $m$, the performance of both algorithms keeps growing. This is because as the task number increases, the task reward will also increase, but for a fixed number of agents the total communication cost is limited, thus the total system profit will keep rising.

Figure 9 shows the average encoding revision time of the two algorithms with regard to different $m$ under two experimental settings respectively, showing that it is slightly better in the second setting than the first. As $m$ increases, the average encoding revision time grows for both algorithms under the two settings, but with a low growth rate. Generally, our algorithm performs much better than the 
Figure 7. The average encoding revision time of each algorithm with different $n$

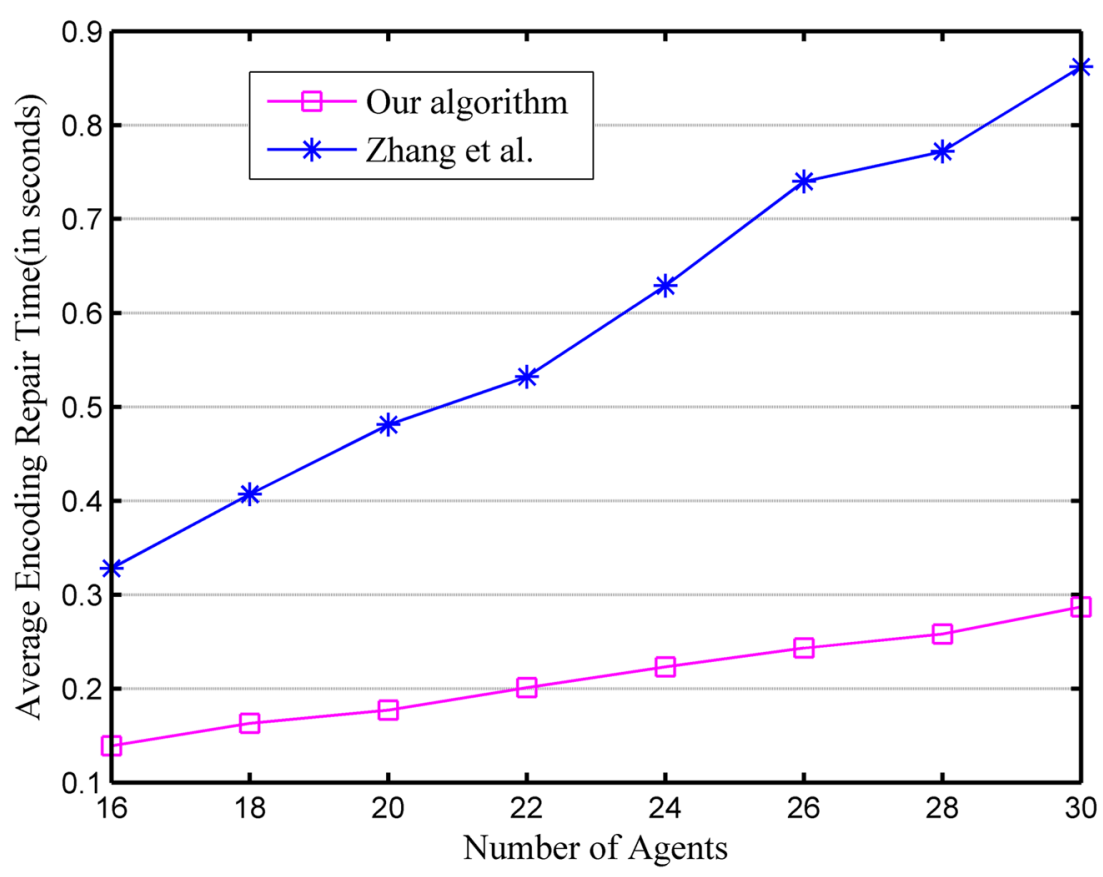

Figure 8. The average total profits of each algorithm with different $m$

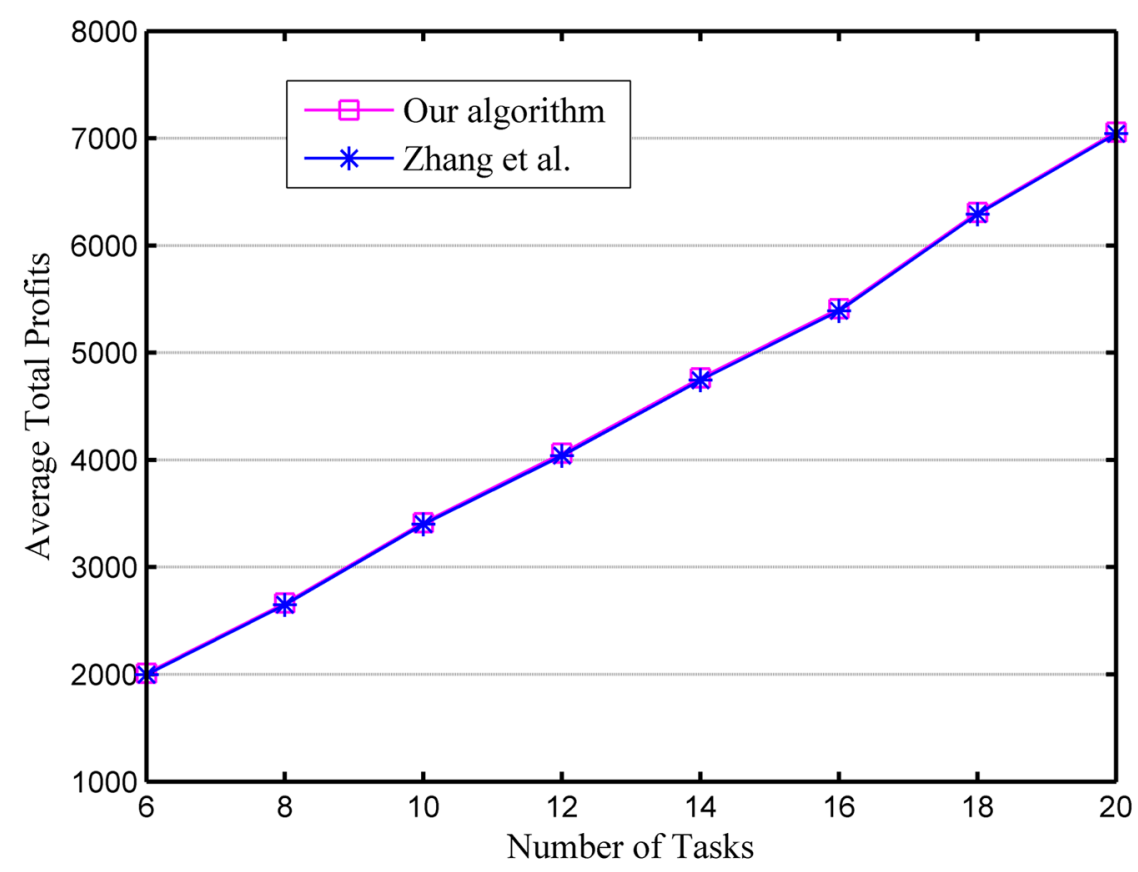


Figure 9. The average encoding revision time of each algorithm with different $m$

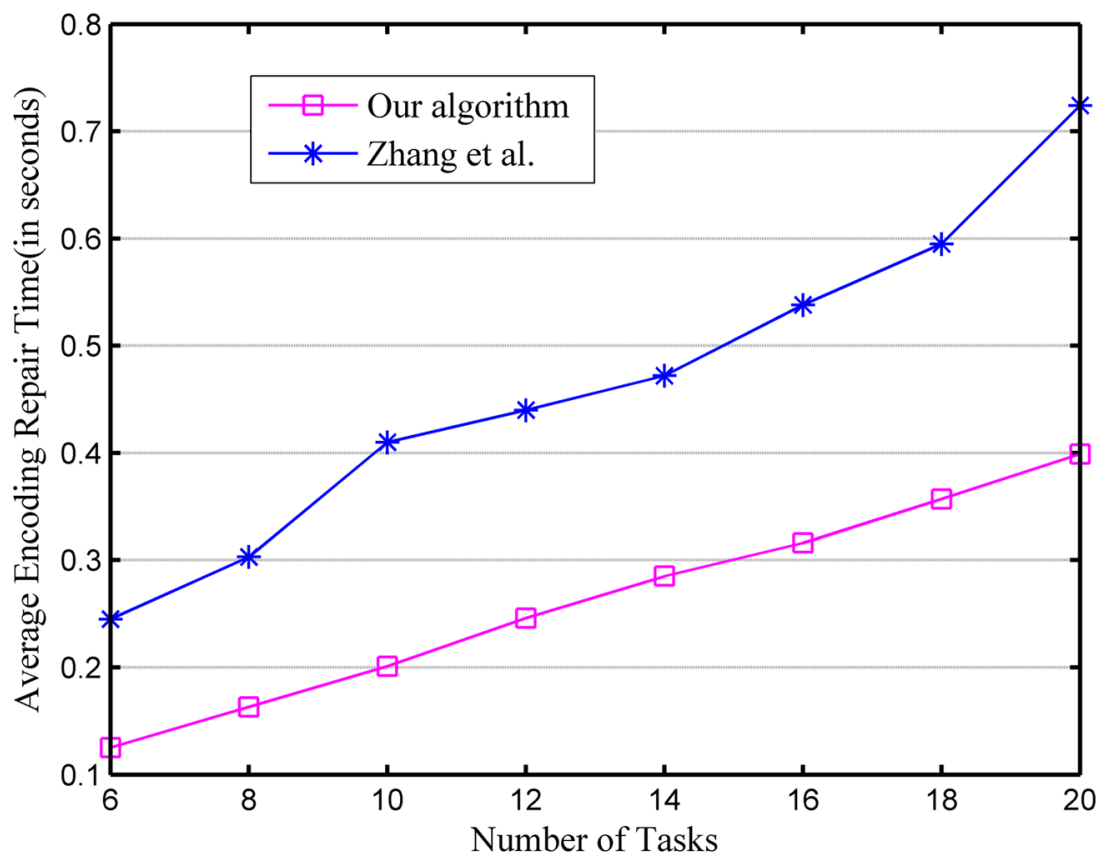

algorithm of Zhang et al. (2011) The experimental results demonstrate that both algorithms are insensitive to the changes of $m$. This is because, when dealing with resource conflicts, the two algorithms focus on checking the columns to calculate the rest resources of the agent and perform fewer revision operations on the rows.

(3) Variation in the number of resource types

In the end, we evaluate the performance of the two algorithms with respect to the number of resource types changing from 2 to 16 , at the number of agents $n=10$ and the number of tasks $m=4$. Figure 10 shows the average total profits of the two algorithms with different $r$ under the two experimental settings. As shown in the figures, under either setting, for each algorithm, the total profit is decreasing, till reaching 0 . This is because as $r$ increases, the resource cost keeps growing, but the task reward is unchanged and also the total communication cost between agents, thus the total profit of the coalition will shrink. And when the resource cost increases to some degree, namely, the task reward is less than or equal to the sum of the resource costs and the communication costs of all the agents in the coalition, the system profit would be 0 . And, although under both settings, the two algorithms show little difference, ours is always slightly better than the other.

Figure 11 lists the average encoding revision time of the two algorithms with different $r$ under two experimental settings, from which we can see little difference and only a slight advantage in the second setting over in the first. Experimental results demonstrate that the two algorithms are both sensitive to the changes of $r$, and with the increasing of $r$, their average encoding revision time would increase under both settings. Generally, our algorithm is superior to that of Zhang et al. (2011), and this superiority becomes more obvious as $r$ continues increasing. This is because in both settings, the algorithm of Zhang et al. (2011) needs to visit every " 1 " in all the rows to calculate $W_{j}$, and to 
Figure 10. The average total profits of each algorithm with different $r$

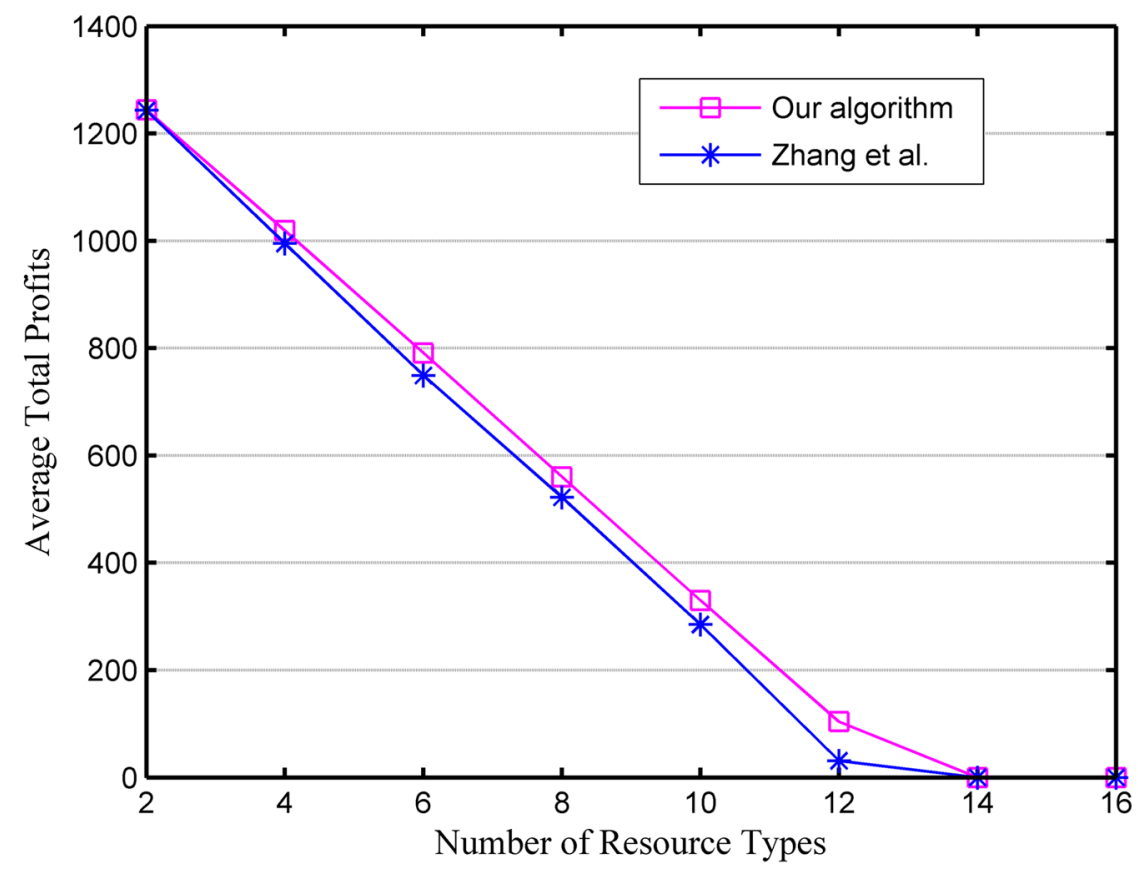

Figure 11. The average encoding revision time of each algorithm with different $r$

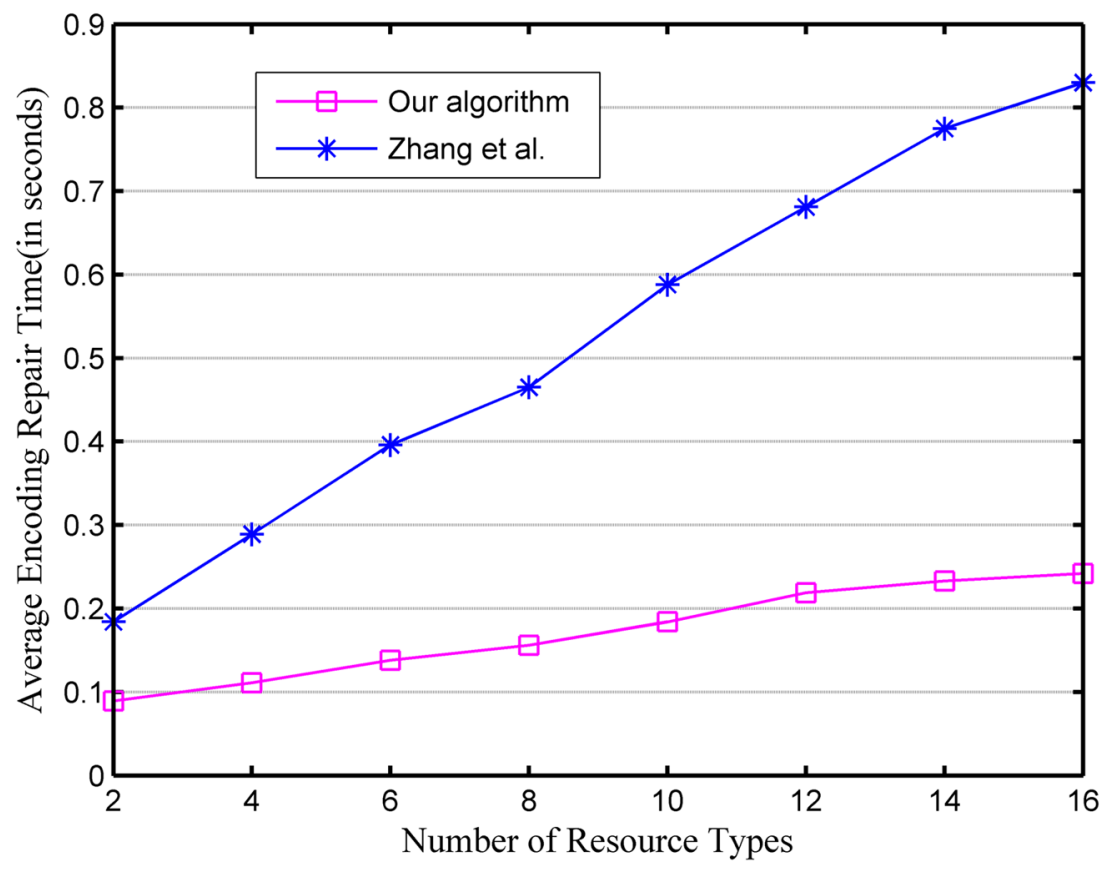


check the next column, it needs to update $C_{i}$ and $B_{C_{i}}$ in this row, which means lots of redundant operations and therefore increased time costs.

In conclusion, the total profits and the encoding revision time obtained by the algorithm in this paper under the two experimental settings are better than those obtained by the algorithm of Zhang et al. (2011). The $n$ and $r$ parameters have obvious influence on the algorithm of Zhang et al. (2011), and the $m$ parameter is relatively insensitive to its influence. Therefore, the algorithm of Zhang et al. (2011) is only suitable for coalition formation problems with relatively small number of agents and resource types. The algorithm in this paper is not sensitive to $m, n$ and $r$, but the total profits obtained by the algorithm in this paper in the first experimental setting, is significantly better than that of Zhang et al. (2011), and there is little difference in the second experimental setting, while the encoding revision time is better than that of Zhang et al. (2011) in both experimental environments. This shows that the algorithm of Zhang et al. (2011) is only applicable to situations where agent resources are very sufficient, while the algorithm in this paper performs well in the two experimental settings and is suitable for various situations.

\section{CONCLUSION AND FUTURE WORK}

In this paper, we propose a new encoding revision algorithm that can revise an invalid encoding into a valid one, randomly select a row to be revised, and then randomly select other unselected rows for encoding revision until all the rows are checked. We solve the overlapping coalition based on the binary difference evolution algorithm and compare with (Zhang et al., 2011). The results show that the proposed algorithm can obtain a better value of overlapping coalition within a shorter encoding revision time. In the future research, we will study the model of overlapping coalition formation and algorithm design when the resources owned by all the agents are less than those required by all the tasks. 


\section{REFERENCES}

Bristow, M., Fang, L., \& Hipel, K. W. (2014). Agent-based modeling of competitive and cooperative behavior under conflict. IEEE Transactions on Systems, Man, and Cybernetics. Systems, 44(7), 834-850. doi:10.1109/ TSMC.2013.2282314

Cavalini, A. A. Jr, Lobato, F. S., Koroishi, E. H., \& Steffen, V. Jr. (2016). Model updating of a rotating machine using the self-adaptive Differential Evolution algorithm. Inverse Problems in Science and Engineering, 24(3), 504-523. doi:10.1080/17415977.2015.1047364

Chalkiadakis, G., Elkind, E., Markakis, E., Polukarov, M., \& Jennings, N. R. (2010). Cooperative games with overlapping coalitions. Journal of Artificial Intelligence Research, 39(1), 179-216. doi:10.1613/jair.3075

Dhaliwal, K. K. (2017). Integrated Cat Swarm Optimization and Differential Evolution Algorithm for Optimal IIR Filter Design in Multi-Objective Framework. Circuits Systems and Signal Processing, 36(1), $270-296$.

Duarte, P. B. F., Fadlullah, Z. M., Vasilakos, A. V., \& Kato, N. (2012). On the partially overlapped channel assignment on wireless mesh network backbone: A game theoretic approach. IEEE Journal on Selected Areas in Communications, 30(1), 119-127. doi:10.1109/JSAC.2012.120111

Dutta, A., Dasgupta, P., Baca, J., \& Nelson, C. (2014). SearchUCSG: A fast coalition structure search algorithm for modular robot reconfiguration under uncertainty. Robotica, 32(2), 225-244. doi:10.1017/S0263574714000095

uerrero, J., \& Oliver, G. (2012). Multi-robot coalition formation in real-time scenarios. Robotics and Autonomous Systems, 60(10), 1295-1307. doi:10.1016/j.robot.2012.06.004

He, W., Chen, G., Han, Q., \& Qian, F. (2017). Network-based leader-following consensus of nonlinear multi-agent systems via distributed impulsive control. Information Sciences, 380(20), 145-158. doi:10.1016/j.ins.2015.06.005

He, Z., Cheng, T. C. E., Dong, J., \& Wang, S. (2014). Evolutionary location and pricing strategies in competitive hierarchical distribution systems:A spatial agent-based model. IEEE Transactions on Systems, Man, and Cybernetics. Systems, 44(7), 822-833. doi:10.1109/TSMC.2013.2290506

Huang, B., Gao, C., \& Chen, L. (2011). Partner selection in a virtual enterprise under uncertain information about candidates. Expert Systems with Applications, 38(9), 11305-11310. doi:10.1016/j.eswa.2011.02.180

Ke, G. Y., Bookbinder, J. H., \& Kilgour, D. M. (2014). Coordination of transportation and quantity discount decisions, with coalition formation. International Journal of Production Research, 52(17), 5115-5130. doi:10 $.1080 / 00207543.2014 .899716$

Khan, M. A., Tembine, H., \& Vasilakos, A. V. (2012). Game dynamics and cost of learning in heterogeneous 4G networks. IEEE Journal on Selected Areas in Communications, 30(1), 198-213. doi:10.1109/JSAC.2012.120118

Kulkarni, A. J., \& Tai, K. (2010). Probability collectives: A multi-agent approach for solving combinatorial optimization problems. Applied Soft Computing, 10(3), 759-771. doi:10.1016/j.asoc.2009.09.006

Liao, S. S., Zhang, J. D., Lau, R., \& Wu, T. (2014). Coalition formation based on marginal contributions and the Markov process. Decision Support Systems, 57(1), 355-363. doi:10.1016/j.dss.2013.09.019

Lin, C. F., \& Hu, S. L. (2007). Multi-task overlapping coalition parallel formation algorithm. Proc. of the 6th Int. Conf. on Autonomous Agents and Multi-agent Systems, 1260-1262.

Liu, A., Li, Q., Huang, L., Ying, S., \& Xiao, M. (2013). Coalitional game for community-based autonomous web services cooperation. IEEE Transactions on Services Computing, 6(3), 387-399. doi:10.1109/TSC.2012.12

Loia, V., \& Vaccaro, A. (2014). Decentralized economic dispatch in smart grids by self-organizing dynamic agents. IEEE Transactions on Systems, Man, and Cybernetics. Systems, 44(4), 397-408. doi:10.1109/ TSMC.2013.2258909

Panigrahi, S. (2017). A Novel Hybrid Chemical Reaction Optimization Algorithm with Adaptive Differential Evolution Mutation Strategies. for Higher Order Neural Network Training. The International Arab Journal of Information Technology, 14(1), 18-25. 
Rahwan, T., Michalak, T., Wooldridge, M., \& Jennings, N. R. (2012). Anytime coalition structure generation in multi-agent systems with positive or negative externalities. Artificial Intelligence, 186(3), 95-122. doi:10.1016/j. artint.2012.03.007

Schreiber, C., \& Carley, K. M. (2013). Validating agent interactions in construct against empirical communication networks using the calibrated grounding technique. IEEE Transactions on Systems, Man, and Cybernetics. Systems, 43(1), 208-214. doi:10.1109/TSMCA.2012.2192104

Sen, S., \& Dutta, P. (2000). Searching for optimal coalition structures. Proc. of the 4th Int. Conf. on Multi-agent Syst., 286-292.

Seow, K. T., \& Sim, K. M. (2007). Coalition formation for resource collocation using BDI assignment agents. IEEE Trans on Systems, Man and Cybernetics Part C-Applications and Reviews, 37(4), 682-693. doi:10.1109/ TSMCC.2007.897490

Service, T. C., \& Adams, J. A. (2011a). Coalition formation for task allocation: Theory and algorithms. Autonomous Agents and Multi-Agent Systems, 22(2), 225-248. doi:10.1007/s10458-010-9123-8

Service, T. C., \& Adams, J. A. (2011b). Constant factor approximation algorithms for coalition structure generation. Autonom Agents and Multi-agent Systems, 23(1), 1-17. doi:10.1007/s10458-010-9124-7

Shehory, O., \& Kraus, S. (1996). Formation of overlapping coalitions for precedence-ordered task-execution. Proc. of the 2th Int. Conf. on Multi-agent Syst., 330-337.

Storn, R. (1995). Kenneth Price, Differential Evolution-A Simple and Efficient Adaptive Scheme for Global Optimization over Continuous Spaces, University of California. ICSI.

Storn, R., \& Price, K. (1997). Differential evolution: A simple and efficient heuristic for global optimization over continuous space. Journal of Global Optimization, 11(4), 341-359. doi:10.1023/A:1008202821328

Vig, L., \& Adams, J. A. (2006). Multi-robot coalition formation. IEEE Transactions on Robotics, 22(4), 637-649. doi:10.1109/TRO.2006.878948

Voice, T. D., Polukarov, M., \& Jennings, N. R. (2014). Coalition structure generation over graphs. Journal of Artificial Intelligence Research, 45(1), 165-196.

Wang, C. Y., Ko, C. H., Wei, H. Y., \& Vasilakos, A. V. (2016). A boting-based Femtocell downlink cell-breathing control mechanism. IEEE/ACM Transactions on Networking, 24(1), 85-98. doi:10.1109/TNET.2014.2357498

Wang, T., Song, L., Han, Z., \& Saad, W. (2014). Distributed Cooperative Sensing in Cognitive Radio Networks: An Overlapping Coalition Formation Approach. IEEE Transactions on Communications, 62(9), 3144-3160. doi:10.1109/TCOMM.2014.2350503

Wilson, B., \& desJardins, M. (2007). Forming stable, overlapping coalitions in an open multi-agent system. Technical Report from the AAAI Fall Symposium on Regarding the "Intelligence" in Distributed Intell. Syst., Arlington, VA.

Xiao, Y., Chen, K. C., Yuen, C., Han, Z., \& DaSilva, L. A. (2015). A Bayesian overlapping coalition formation game for device-to-device spectrum sharing in cellular networks. IEEE Transactions on Wireless Communications, 14(7), 4034-4051. doi:10.1109/TWC.2015.2416178

Xu, J., \& Li, W. (2008). Solution of overlapping coalition formation based on discrete particle swarm optimization. Proceedings of the International Conference on Wireless Communications, Networking and Mobile Computing, $1-4$.

Xu, S., Xia, C., \& Kwak, K. (2016). Overlapping coalition formation games based interference coordination for D2D underlaying LTE-A networks. Journal of Electronics and Communications, 70(2), 204-209. doi:10.1016/j. aeue.2015.10.007

Yang, J., \& Luo, Z. (2007). Coalition formation mechanism in multi-agent systems based on genetic algorithms. Applied Soft Computing, 7(2), 561-568. doi:10.1016/j.asoc.2006.04.004

Ye, D., Zhang, M., \& Sutanto, D. (2013). Self-adaptation-based dynamic coalition formation in a distributed agent network: A mechanism and a brief survey. IEEE Transactions on Parallel and Distributed Systems, 24(5), 1042-1051. doi:10.1109/TPDS.2012.213 
Ye, D., Zhang, M., \& Sutanto, D. (2015). Decentralised dispatch of distributed energy resources in smart grids via multi-agent coalition formation. Journal of Parallel and Distributed Computing, 83(4), 30-43. doi:10.1016/j. jpdc.2015.04.004

Yu, X. B. (2017). Disaster prediction model based on support vector machine for regression and improved differential evolution. Natural Hazards, 85(2), 959-976. doi:10.1007/s11069-016-2613-5

Zhang, G., Jiang, J., Lu, C., Su, Z., Fang, H., \& Liu, Y. (2011). A revision algorithm for invalid encodings in concurrent formation of overlapping coalitions. Applied Soft Computing, 11(2), 2164-2172. doi:10.1016/j. asoc.2010.07.015

Zhang, G. F., Jiang, J. G., Su, Z., Qi, M., \& Fang, H. (2010). Searching for overlapping coalitions in multiple virtual organizations. Information Sciences, 180(17), 3140-3156. doi:10.1016/j.ins.2010.04.028

Zhao, Z., Yang, J., Hu, Z., \& Che, H. (2015). A Differential Evolution algorithm with self-adaptive strategy and control parameters based on symmetric Latin hypercube design for unconstrained. European Journal of Operational Research, 250(1), 30-45. doi:10.1016/j.ejor.2015.10.043

Haixia Gui received the B.S. and M.S. degrees in Technology, Huainan, China, in 2001 and 2008, respectively. And she received the Ph.D. degree in computer science from Hefei University of Technology, Hefei, China, in 2018. She is currently an associate professor of the School of Economics and Management Engineering, Anhui University of Science and Technology, Huainan, China. Her current research interests include multi-agent systems, evolutionary computation, and multi-objective optimization.

Banglei Zhao received the B.S. degree in accounting from Dezhou University, Dezhou, China, in 2017. He is currently pursuing the M.S. degree in logistics engineering at School of Economy and Management, Anhui university of science and technology, China. His current research interests include cold chain logistics and path optimization. $\mathrm{He}$ is a member of the China society of logistics.

Huizong Li received the HP.D. degree in computer application technology from Hefei University of Technology, Hefei, China, in 2016. He is currently an Associate Professor with Nanyang Normal University of China. He has authored or coauthored more than 30 articles. His current research interests include the areas of machine learning, big data and big knowledge management, social network and intelligent information processing. He has received one Second-Class Science and Technology Progress Award from Ministry of Education (MOE) of China.

Wanliu Che is currently pursuing a bachelor's degree in electronic commerce with Anhui University of Science and Technology. His current research focus is data mining. 